Comparaison générique vs comparaison prototypique. Ou de l'évaluation « con-forme » par concept-mesure à l'évaluation instituante ou « analogique » par concept-matrice

\title{
Alain Papaux
}

\section{OpenEdition}

\section{Journals}

Édition électronique

URL : http://journals.openedition.org/ress/198

DOI : $10.4000 /$ ress. 198

ISSN : $1663-4446$

Éditeur

Librairie Droz

Édition imprimée

Date de publication : 2 juillet 2007

Pagination : 115-141

ISBN : 2-600-01170-9

ISSN : 0048-8046

\section{Référence électronique}

Alain Papaux, «Comparaison générique vs comparaison prototypique. Ou de l'évaluation « con-

forme » par concept-mesure à l'évaluation instituante ou « analogique » par concept-matrice », Revue européenne des sciences sociales [En ligne], XLV-138 | 2007, mis en ligne le 01 juillet 2010, consulté le 19 avril 2019. URL : http://journals.openedition.org/ress/198 ; DOI : 10.4000/ress. 198 
Alain PAPAUX

\title{
COMPARAISON GÉNÉRIQUE VS COMPARAISON PROTOTYPIQUE ou de l'évaluation «con-forme» par concept-mesure à l'évaluation instituante ou «analogique» par concept-matrice
}

\author{
1. OUVERTURE \\ UNE MESURE DES PLUS CONCRÈTES : \\ LE CALCUL DES DÉLAIS
}

Parmi les considérations les plus concrètes qu'ont à traiter les juristes, on rangera sans conteste le calcul des délais, dont nous allons montrer, qu'à peine entrepris, il en appelle à l'équité, alors même que l'on croyait, enfin, détenir quelque notion juridique qui fût parfaitement épuisée par une réalité mathématique semblant confiner à une pure et simple concaténation, un décompte mécanique s'opérant à l'abri de toute intervention de la subjectivité

Ainsi, en va-t-il de la loi helvétique sur l'aide aux victimes d'infractions (LAVI) laquelle, pour fixer schématiquement le cadre de cette ouverture, prévoit la substitution, partielle, de l'Etat à l'auteur du crime ou du délit portant sur l'indemnisation et la réparation morale pour le dommage subi quand l'auteur demeure inconnu ou se révèle insolvable. Pour obtenir le bénéfice de cette loi, la victime doit déposer une demande d'indemnisation LAVI, dans un délai deux ans à compter de la date de l'infraction. L'art. 16 arrêtant cette modalité, ne devrait-il pas emporter l'adhésion de tous les juristes, jusqu'à convaincre les plus dubitatifs de la réussite même du projet d'une «Rechtswissenschaft» puisqu'enfin le droit aurait un domaine intégralement mathématisé: les délais et leur calculs. Là, mieux que partout ailleurs en droit, semble valoir la prosopopée qui garantit aux juristes sa parfaite neutralité: «la loi dit que...». Ce calcul n'est-il pas aisé? Un simple calendrier n'y suffirait-il point?

Mais le Tribunal fédéral ${ }^{1}$ aura tôt fait de nuancer cette affirmation, estimant que la situation particulière des victimes induit un devoir accru d'informations de la part de l'Etat et, partant, revisite la logique plate du calendrier en en abandonnant une lecture quantitative au profit d'une interprétation qualitative. Aussi ce délai de deux ans se décompose-t-il en une regula, soit une règle, une régularité, à même de saisir «les cas les plus fréquents », à savoir un décompte classique de deux ans à partir du point de départ arrêté dans la loi, cette perspective faisant fond sur une dimension plus englobante, excédant le cadre strict (formalisme) de la loi: l'équité. Comme

1 En particulier ATF 123 II 241 (ATF=Arrêts du Tribunal fédéral [suisse]) rendu le 3 juillet 1997. 
pour toute règle juridique, somme toute, son exercice concret - comprenant non seulement son sens technique d' «application» mais encore et surtout un sens «métaphysique », ultime, s'exprimant en termes de fin, de destinée de la norme - se trouve implicitement dans la dépendance de considérations d'équité sans la référence auxquelles il ne serait plus possible de parler de justice c'est-à-dire de jus, droit en latin ${ }^{2}$. Le ressort de la décision en cause en matière LAVI se trouve fort bien ramassé dans le chapeau de la Cour suprême, relevant que «...le devoir d'information des autorités policières et judiciaires a pour corollaire que la victime ne doit subir aucun préjudice d'un défaut d'information qui l'a empêchée d'agir à temps sans sa faute. Cas exceptionnel où l'équité commande de ne pas opposer à la victime le délai de péremption de deux ans fixé à l'art. 16 al. 3 LAVI (consid. 3).»

Par où l'on voit que la loi, au sens le plus formel, et quand bien même semblet-elle prima facie ne nécessiter aucun recours à l'interprétation, s'avère être une directive, une orientation, pour qui considère cette dimension globale, englobante, de l'équité. Ce changement de perspective dans la compréhension de la loi, plus exactement de sa signification, présente son relief le plus marqué dans l'explicitation des critères de «juridicité» qui structurent chacune des dimensions relevées, celle formelle et celle matérielle, métaphysique.

Nous montrerons qu'à la conception formelle de la loi (légalisme) correspond la logique classique genrelespèces, connue sous la figure de l'arbre de Porphyre. A la conception «matérielle» de la loi, celle mettant l'accent sur les finalités et parmi celles-ci, en dernier lieu, l'équité, répond la logique systémique, rhizomique ou encore abductive de la dialectique type-occurrences, ces dernières rétroagissant sur le type ou catégorie légale.

A la dimension formelle ou légaliste, correspond le critère de la conformité, très exactement «con-formité», soit présenter la même forme $e^{3}$, ne rendant pertinent dans le cas que ce qui s'y trouve à l'identique par rapport à l'étalon «loi » ou «catégorie légale». De la sorte s'instaure un rapport mimétique trait pour trait, lequel présuppose une sémiotique fondée sur des primitifs ${ }^{4}$ que permettrait d'identifier une analyse au sens strict: réduction d'un phénomène à ses composantes les plus petites et telle que leur compréhension permet de rendre compte, par simple somme, du niveau hiérarchique supérieur, à savoir celui du phénomène en question. Cette analyse est dite componentielle dans une certaine linguistique. Il en résultera un mode de comparaison statique, de simple subsomption de l'espèce (cas) sous le genre, soit une comparaison générique typique d'une métaphysique analytique ou réductionniste.

2 Le Tribunal fédéral a confirmé cette assertion à la faveur de l'explicitation d'une méthode d'interprétation que l'on pensait être parfaitement objective, soit à l'abri de toute considération d'équité: la méthode littérale; voir infra, Chapitre 4, Paragraphe 1.

3 Il s'agit bien ici de forme-eidos (essence ou principe de toute réalité ou ce qui fait qu'une chose est ce qu'elle est) et non de la forme-morphe (la forme sensible, l'apparence, le contour extérieur). C'est le propre du positivisme juridique dans sa version la plus dure, à savoir le légalisme, version toute théorique évidemment, que de confondre dans le droit posé (jus positum, droit posé) ces deux acceptions de «forme».

4 U. Eco, Les limites de l'interprétation, Le livre de poche, Paris, 1994, 360, les définit: «expressions métalinguistiques qui ne sont pas des mots en soi et qui ne nécessitent aucune interprétation ultérieure.» 
$\mathrm{Au}$ contraire, pour qui adopte la conception large, englobante, du droit ou juste, la comparaison avec l'entité de référence, à savoir la «catégorie légale», ne procède plus d'une mesure exacte, quantifiée, à tout le moins réduite à une identité trait pour trait, laquelle supprime la singularité du cas particulier à considérer - mais s'engage dans des processus dynamiques et très complexes de pesée des intérêts et d'abductions, recherchant la «bonne mesure », la juste mesure. Cet univers quitte une métaphysique analytique pour une philosophie opérant principalement et principiellement par analogies. La loi ne se reçoit dès lors plus comme un étalon qu'il suffirait de plaquer sur le cas pour en identifier ce qui est juridiquement pertinent et en écarter ce qui «dépasse »; elle devient un raccourci cognitif à l'instar de la peinture médiévale toute pénétrée de préoccupations pédagogiques ramassant par symbolisation les épisodes exemplaires et les affirmations cruciales de la religion chrétienne à destination des croyants analphabets. Les juristes, lettrés par profession, recourent à des raccourcis infiniment plus éthérés, à peine exhibés, densément sédimentés dans leur culture juridique (près de dix ans de formation) et qui, dans leur ressort, procèdent d'une manière analogue aux tableaux médiévaux élaborés en superbes «machines » mnémotechniques à suivre l'historien de l'art Daniel Arasse.

\section{PROPÉDEUTIQUE LA RÉFLEXION SUR LA MESURE COMME PARADIGME DES CHANGEMENTS DANS L'APPRÉHENSION DU NORMATIF}

Dans l'Antiquité qui nous est connue par les figures nodales de la philosophie, la mesure s'entend principiellement de «bonne mesure», le fameux «juste milieu» attribué à Aristote et trop souvent compris comme moyenne au lieu d'optimum ou médiété : un sommet ou summum entre deux extrêmes, le «trop » et le «trop peu». Cette mécompréhension révèle déjà le tour d'esprit quantificateur, «mathématisé » de la pensée moderne, lequel requiert une pensée de l'illimitation, de l'infini.

On ne saurait trop souligner l'importance de cette illimitation, de l'hybris, de la démesure dans la conception du «vivre ensemble» selon une expression moderne aspirant à l'originalité. Cette illimitation apparaît comme une marque de la modernité, verrons-nous. A tout le moins en va-t-il ainsi de l'illimitation entendue au sens de sans limite, sans terme, sans frontière. Cette illimitation semble en effet étrangère à l'Antiquité, laquelle, pour commencer par le savoir architectonique, confinait la politique à la polis, l'arrêtait aux murailles de la cité, plaçant les sciences c'est-à-dire l'étude de la nature proprement hors les murs. Le Moyen Age fut lui aussi tout pénétré de cette finitude, expressément revendiquée par Thomas d'Aquin, lequel, dans la ligne de son maître Aristote, affirmait en substance que tout «reçu $»$ l'est selon les modes du « recevant $»^{5}$.

5 Formule répandue dans les disputes médiévales, voir J. Tricot, in Aristote, Métaphysique, V, 12, 284 note 3 et IX, 1, note 3, 484 de la traduction de Tricot, 2 vol., Vrin, Paris, 1991 et 1986. On trouve des formules analogues sous la plume de Thomas d'Aquin: secundum modum intelligibilem 
On relèvera que le «bien» de l'expression «bien vivre ensemble» ne traduit pas simplement une nuance mais signe une conception fondamentalement différente du politique, se contentant, dans l'acception moderne, de l'articulation d'individus se percevant comme des monades, complets en eux-mêmes, de simples atomes sociétaux du point de vue du collectif. Leur mode de co-présence au monde serait ainsi de l'ordre de la juxtaposition. On se rappellera que pour les Anciens, en revanche, le «bien vivre ensemble» porte loin au-delà d'une simple articulation d'atomes sociétaux pour se concentrer dans l'assomption du bonheur de chacun dans le bonheur (ou harmonie) de la cité. L'individu comme individu n'y avait point place, la personne se pensant toujours déjà comme citoyen, à l'exception notable des Stoïciens et des Cyniques. Dans cette conception essentiellement collective, la cité n'absorbe pas le citoyen mais tend à le «par-faire» (en théorie du moins), à le faire «être» en toutes ses parts, à l'achever, en l'assumant sur le plan politique en qualité de citoyen précisément. La cité se déploie comme le lieu où le citoyen est le plus lui-même en tant que la présence de l'Autre est constitutive de sa propre identité ${ }^{6}$. Il y réalise ou actualise ses potentialités ou puissances les plus profondes, sa fin la plus intime, à tout dire son essence d'homme. La mesure, plus exactement la bonne mesure, s'éprouve comme la relation mesurée à l'autre.

Comprenant de la sorte cette relation mesurée, on se gardera de la recevoir selon les oppositions binaires substance ou accident, essence ou existence, mais bien plutôt la considérera-t-on comme une tension ${ }^{7}$ que traduit le couple «puissance/acte» théorisé par Aristote pour tenter de caractériser le devenir, le changement, en bref la dynamique de la vie ${ }^{8}$. La «puissance» dont il s'agit ${ }^{9}$ renvoie à la notion de «potentialité » tirée de potestas soit, en latin, la puissance, le pouvoir.

On retrouve ici l'opposition entre deux visions du monde, qui donnera aussi une distinction entre deux types de droit: d'une part un réel conçu selon la causalité efficiente, monde de forces au sens large de force-potestas (comprenant aussi bien les forces ou puissances physiques que le pouvoir-puissance politique), éla-

(Somme Théologique I, q. 14. art. 5), secundum esse intelligibile (Somme Théologique I, q. 15, art.1). Leur compréhension en appelle à la distinction entre les perspectives d'intelligibilité du réel selon l'essence ou «quiddité » et selon l'existence ou exercice, et plus particulièrement l'assomption de l'exercice non comme «accident» (au sens philosophique) mais comme perfection de l'essence; «l'acte du senti et [l'acte] de la sensation est le même et unique», «l'âme est d'une certaine manière toutes choses; les êtres sont en effet soit sensibles, soit intelligibles, et la science est d'une certaine manière les objets de science, la sensation les sensibles » (De anima, 425 b 25-27; 431 b 21-23)» traduit A. de Muralt, in Néoplatonisme et aristotélisme dans la métaphysique médiévale, Vrin, Paris, 1995, 114.

6 On reconnaît ici la définition canonique de l'homme par Aristote: zoon politikon, reprise par les sociologues contemporains parlant d'animal politique, manquant par là une partie de la portée de l'expression aristotélicienne, mieux rendue par «vivant politique» pour bien marquer qu'il ne s'agit pas d'une essence éthérée de l'homme mais de la description de sa nature concrète, pragmatique (sachant que pragma/pragmata signifie «existant» en grec).

7 Voir supra note 5.

8 Nous ne saurions toutefois nourrir la moindre nostalgie à l'endroit du modèle athénien de la démocratie, système politique dans lequel, on l'oublie trop souvent de nos jours quand on affirme vouloir s'en inspirer, tout le monde surveillait chacun.

9 Cette puissance n'est pas sans lien avec la notion de potentiel abordé dans l'article de Françoise Schenk, dans ce volume. 
boré sur le modèle de la physique (le parangon épistémologique de la modernité); d'autre part un réel organisé principiellement autour de la causalité finale, des fins, des buts.

L'illimitation de principe qui caractérise la modernité ruine la notion de «bonne mesure »- notion qualitative -, reflétant une pensée toute concentrée sur le modèle scientifique du monde, celui de la physique mathématisée.

Sous l'égide de ce modèle, porté expressément par Galilée et Descartes dans l'entreprise de mathématisation du monde et répercuté en droit par et dans l'Ecole du droit naturel moderne (expressément sous la plume d'un Puffendorf, par exemple), la mesure s'installe résolument dans une acception quantitative, la dimension du qualitatif devenant accidentelle et marginale. On se souviendra à ce titre de l'entreprise cartésienne de réfutation de la physique qualitative aristotélicienne $^{10}$. La relégation de l'interprétation par les fins dite «méthode téléologique» au rang de moyen dernier (ultima ratio) dans la théorie classique de l'interprétation juridique atteste l'effacement des fins, la médiocre pertinence de la finalité, du but, dans la dogmatique juridique.

Le normatif suivra le même renversement de la qualité à la quantité, parfaitement perceptible avec le substitut normatif de la quantité, savoir la «con-formité » - c'est le thème central de cette contribution -, figure nodale du positivisme juridique dans sa version légaliste, lequel traduit emblématiquement la pénétration des esprits juridiques par le scientisme.

La modification sémantique, et plus globalement sémiotique, de la «mesure» exprime synthétiquement l'état d'esprit dans lequel la normativité est conçue et plus spécifiquement encore la manière dont la loi saisit les cas, les occurrences du «réel».

Il y a, proposons-nous, une distinction fondamentale à opérer entre, d'une part, une «comparaison générique» (genres/espèces) entre catégorie légale et «espèce à classer» (cas), laquelle correspond à une logique de la conformité, substitut scientiste de la mesure quantitative dans le domaine normatif (Chapitre 3), et, d'autre part, une «comparaison prototypique» entre catégorie légale conçue comme matrice (type) et «occurrence à juger», régie par une logique opérant via des raisonnements par analogie, à même d'épouser (plus ou moins fidèlement) le continuum du réel comme nous tenterons de le montrer (Chapitre 4) à la faveur de l'exemple d'une pancarte interdisant la présence de chiens sur les quais d'une gare: quid de loups, d'ours ou de vaches?

Nous aimerions entreprendre ces deux types de comparaison in situ, dans le lieu naturel du «normatif vivant», à savoir son application à des cas concrets, la pratique, en bref la casuistique. Si la destination du normatif est l'application, il faudra alors se dévêtir des modes de pensée de l'idéalisme, lequel s'intéresse en première ligne, voire exclusivement, à l'Idée de normatif, à sa «forme» (d'où «con-formité») au sens premier de eidos (en grec, qui donnera idée et idéalisme)

10 Descartes ne défendit pas pour autant une physique fondée sur les forces, les estimant participer davantage de quelque puissance occulte donnant bien trop dans une conception encore toute superstitieuse de la nature. Il avança une explication par tourbillons, quelque peu amphigourique. Par sa double appartenance culturelle, à l'alchimie et à la science classique, Newton put surmonter les réticences cartésiennes à l'égard des «forces», en acceptant l'hypothèse des forces et en l'étendant à l'univers entier, le microcosme ne faisant que refléter le macrocosme pour l'alchimiste. 
et qui désigne pour référentiel d'un tel type de pensée l'essence par contraste avec l'existence. La (ou les) signification théorique de la règle règne en maître, au point d'éclipser son sens pratique, sa façon pragmatique de «parler» dans le cas concret, avec toutes les rétroactions que pareille considération du juste incarné dans telle situation précise donne à voir.

\section{LA COMPARAISON GÉNÉRIQUE OU LA MESURE COMME «CON-FORMITÉ»}

Les philosophes non juristes s'occupant de théorie du droit élisent souvent le droit pénal pour modèle de leurs analyses, son appréhension par les laïcs au sens de non-juristes paraissant la plus simple et l'application à portée d'un esprit non rompu aux finesses quasi infinies des catégorisations juridiques. Le modèle pénal affermissait la conception abstraite du droit en ce qu'il manifestait (surtout à l'époque, $18^{\mathrm{e}}$ et $19^{\mathrm{e}}$ siècles), de la manière la plus explicite la verticalité descendante - Etat en haut, coupable en bas -, épousant de la sorte l'inclination naturelle de la pensée idéaliste suivant le parangon platonicien - l'Idée en haut, au Ciel des Idées, et les cas en bas, tout en bas, au fond de la caverne, ces espèces constituant autant d'accidents du genre (ou essence).

Aussi la philosophie moderne du droit, généralement portée par des non-praticiens, voire des non-juristes, arrête souvent ses analyses à la loi écrite - code -, suspendue dans les airs, mieux, ancrée dans l'éther, ciel pur de tout cas. Il ne s'agit aucunement ici d'écarter de la réflexion juridique les non juristes mais de rendre attentif à l'inadéquation de maintes analyses par oubli de la pratique du droit dans l'appréhension du phénomène juridique. Une conception aussi désincarnée du droit n'est point le fait des seuls non-juristes. Des doctrines entières donnèrent dans cet essentialisme pour des raisons généralement idéologiques: du juge «bouche de la loi » ${ }^{11}$ dans l'Ecole de l'Exégèse faisant suite, en parfaite cohérence, au Code Napoléon, à maints théoriciens contemporains continentaux continuant de soutenir l'existence de méthodes d'interprétation, au rebours du Tribunal fédéral avons-nous vu, méthodes objectives et hiérarchisées, sans pouvoir fournir au reste le critère de cette hiérarchisation. Les travaux de linguistique, de sémiotique, d'herméneutique et de sociologie ont, depuis nombre d'années, fait justice de cette conception désincarnée de l'interprétation. En bref, les juristes euxmêmes arrêtent souvent leur attention à la loi, oubliant qu'elle a pour finalité propre ou fonction première de résoudre des cas concrets: l'application constitue

11 Les juges devaient, imaginait-on, se contenter de «dire» la loi, précisément la redire, et ne pas l'interpréter; en ce sens, ils n'en seraient que la «bouche». Cette formule remonte en vérité aux «ennemis» jurés de l'Ecole de l'Exégèse, les parlements (au sens de tribunaux) d'Ancien Régime, voir M.-F. Renoux-Zagamé, «Le « Royaume de la loi »: équité et rigueur du droit selon la doctrine des parlements de la monarchie», in Justices. Revue générale de droit processuel, Dalloz, Paris, $\mathrm{N}^{\circ} 9$, 1998, 17ss. (passim). Les juges d'Ancien Régime entendaient l'expression «bouche de la loi» de manière analogue mais dans un contexte théologique chrétien au lieu de laïc comme leurs homologues «révolutionnaires », concevant eux aussi la loi de manière quasiment sacrée; mais pour ceux d'Ancien Régime, la loi s'entend en première ligne de celle de dieu dont le règne doit être organisé dans le monde des hommes. 
le terme naturelle, la fin ultime de la loi, laquelle n'est jamais qu'un moyen du droit, comme la coutume, les principes généraux $d u$ droit et les principes généraux de droit international, la jurisprudence et encore la doctrine.

Demeurant volontairement dans l'abstrait, le désincarné, ce type de pensée adoptera pour critère de juridicité - en réalité de simple légalité - la conformité à un texte de loi préétabli selon des procédures formelles à respecter fidèlement. La conformité doit s'entendre à la lettre, comme «con-formité »: rejouer la «forme» soit l'essence, qui n'est autre que la définition formelle de la catégorie légale ramassée dans le texte de loi. La forme devient de la sorte la mesure du réel en droit, son étalon. Le cas n'existe qu'en tant qu'il s'y conforme, l'espèce ne vit qu'en tant que subsumée au genre.

Cette logique descendante de la conformité, cette loi tombée dans le monde pragmatique des situations concrètes comme l'âme chute dans le tombeau du corps, ne procède-t-elle pas d'un esprit platonicien, plus exactement, s'agissant de loi et de commandement, d'un esprit néoplatonicien tel qu'il a pu pénétrer les premiers siècles chrétiens? L'affirmation est plus banale qu'il n'y paraît tant les origines théologiques du modèle moderne de la loi sont manifestes: de la révérence témoignée à l'endroit du texte ${ }^{12}$, en passant par la précellence voire l'exclusivité du droit écrit, du texte, plus exactement du texte de la loi - comme la Bible définie par d'aucuns le Texte des textes -, quasi monopole expressément consacré par la hiérarchie des sources formelles du droit - la loi en tête évidemment -, rappelée en l'art.1 du CCS (Code civil suisse) mais consacrée dans quasi tous les ordres juridiques continentaux. D'où ce constat philosophique: «La définition du Droit est, dans la doctrine dominante, tout inspirée d'une conception théologique de la Loi et marquée de réminiscence du sacré. La loi, parole de Dieu, ou de son substitut laïque, l'Etat. Sacralisation de la Loi, théologie de la Parole qui est portée à élever la parole au-dessus de l'écriture et à considérer que, puisque la loi est parole, elle est - comme la parole de Celui qui l'a proférée - vérité insusceptible de variation dans son expression, ni son interprétation. Ce qui se trouve ainsi fondé, c'est l'univocité de la loi, la rigueur de ses méthodes d'interprétation, le syllogisme implacable qui aboutit à la solution, la seule solution susceptible de s'imposer au sujet de droit $[\ldots] \gg^{13}$.

Où l'on retrouve la loi comme norme, norma en latin, soit ««équerre», et au figuré, le «modèle», la «loi »» ${ }^{14}$. Mais aussi bien la loi comme règle, du «latin regula « règle» servant à mettre d'équerre ${ }^{15}$, vision orthogonale conduisant littéralement à l'orthodoxie, indiquant l'avis ou l'opinion (doxa) droit, la forme (au

12 Attestée par la primauté reconnue à la méthode d'interprétation littérale, quand la texte ne parle pas de lui-même comme dans la figure du juge bouche de la loi ou dans l'expression courante «la loi dit que...». Sur ce dernier point, voir A. Papaux, Introduction à la philosophie du «droit en situation», Bruylant/L.G.D.J/Schulthess, Bruxelles/Paris/Zurich, 2006, 181 ss.

13 G. Timsit, Les noms de la loi, PUF, Paris, 1991, extrait du résumé du quatrième de couverture. Il n'est guère besoin de rappeler que le Decalogos est au principe des normes essentielles, nodales, de tout le droit pénal occidental.

14 Y. Le Roy / M.-B. Schönenberger, Introduction générale au droit suisse, Schulthess, L.G.D.J, Bruylant, Genève, Paris, Bruxelles, 2002, p. 6 [cité Introduction].

15 Ibidem. 
sens d'eidos) à suivre, celle à laquelle l'individu doit se «con-former» strictement, trait par trait, pour demeurer dans la légalité, laquelle a absorbé tout le droit. Dans une conception légaliste, en effet, le juste ne saurait excéder la loi: elle seule le peut dire, suivant l'adage positiviste «juste (bon) parce que prescrit»: le jus positum, le droit positif pose le juste selon la (bonne) volonté du législateur, luimême en théorie parfaitement autonome, «auto-nomos», se donnant à lui-même toute loi, tout contenu: n'est-il pas souverain ? $^{16}$

L'orthodoxie, pour être conservée dans sa perfection, son homogénéité, requiert une logique de strict classement, une opération mécanique de catégorisation de l'espèce sous le genre - classer sous ou «sub-sumer» d'où subsomption -, le cas étant cognitivement rangé sous la catégorie légale qui lui préexiste et qui ne doit varier puisqu'elle seule a été posée (jus positum) par le législateur, partant bénéficie de sa légitimité. Cette dernière ne vient pas à la règle de droit de par l'assomption d'un certain contenu regardé comme particulièrement digne mais du fait qu'elle est posée. D'un mot, la « règle est un étalon permettant de juger une situation ${ }^{17}$, description canonique qui ne contient aucune référence au juste, à un quelconque contenu matériel, en théorie évidemment.

Pour bien servir le projet d'un droit expurgé (donc pur de) de tout jugement de valeurs, condamnant le corps de juristes, via des «théories pures du droit», à ne jamais pouvoir revenir sur les arbitrages de valeurs effectués par le législateur, ceux-ci devaient-il apparaître à ce corps répugnants ou scandaleux, le mode logique du système juridique légaliste doit d'être déductif.

La déduction, clef classique du raisonnement juridique reçue dans la figure du syllogisme, peut se définir comme un développement au sens géométrique du terme, soit une conception analytique du réel comme relevé. Ce devrait être, en strict positivisme juridique, le seul type reconnu d'inférence. Se déployant à partir d'une majeure, à savoir la loi, posée par le législateur sous la forme d'un commandement, passant par la mineure - la situation à juger - pour conclure mécaniquement au dispositif - la sanction -, la déduction juridique prétend opérer selon des nécessités logiques (idéalement, de logique formelle), écartant toute médiation de la subjectivité, du juge notamment. La caractérisation de la majeure du syllogisme en termes de commandement n'est aucunement anodine. Cette nature, sans être jamais explicitée dans le cadre du raisonnement juridique, le fonde pourtant. En effet, le syllogisme doit l'entier de sa force de conviction à son irréprochabilité logique, laquelle n'est acquise que par la qualité de la majeure: elle doit être nécessaire et non contingente pour irradier une pureté d'objectivité dans l'ensemble des pas de l'inférence.

Le monde de la praxis ne disposant pas de telles prémisses, au rebours des sciences mathématiques et naturelles, les juristes modernes, tout fascinés par le

16 A cette aune volontariste, discrétionnaire, on mesure mieux la dimension révolutionnaire de la Déclaration universelle des droits de l'homme au sortir de la seconde guerre mondiale. Elle devenait, à tout le moins symboliquement (avant son relais par divers droits internationaux régionaux plus efficaces parce que dotés de cours et de procédures pour en sanctionner les violations), le modèle, l'étalon des valeurs minimales que doit respecter tout système juridique qui prétend à la qualité d'Etat de droit. Ces valeurs devinrent donc impératives, s'imposant à tout législateur, à tout souverain même.

17 Y. Le Roy / M.-B. Schönenberger, Introduction, 7. 
positivisme scientifique, cherchèrent dans le domaine du normatif quelque analogue de la nécessité. Pareille tâche devait s'avérer des plus ingrates puisque le ressort même de la praxis (éthique, droit et politique) s'entend de la présence principielle d'un choix: l'authentique nécessité supprime le droit puisque le comportement souhaité sera par définition suivi. Ne demeurait que l'instance du commandement dont on pouvait soutenir théoriquement qu'elle ne prêtait le flanc à aucune intervention de la subjectivité, le commandement se voulant univoque et uniforme, la loi ne devant faire acception de personne comme nous le rappelle Dame Justice aux yeux bandés.

Toutefois, démarche proprement linéaire, plate, la déduction, qui a pour corollaire la subsomption et donc le classement en "genre/espèces », ne parvient pas à surmonter la rupture de continuité entre fait et droit. Considéré le dualisme radical sein/sollen, comment la déduction partant du «genre droit» (sollen) pourraitelle parvenir au «genre fait» (sein), comment pourrait-elle assumer un saut qualitatif, un dialogue transgénérique alors même qu'elle ne conjugue que des causes homogènes: «en passant du principe aux conséquences, on ne fait, en un sens, que passer du même au même ${ }^{18}$. L'absence de tout moment de pari ou d'invention condamne la pensée à un enfermement dans la seule textualité, pis dans la seule littéralité: «La doctrine officielle de l'interprétation présente cette activité comme dépourvue de toute dimension créatrice: le texte prédétermine le sens et l'interprétation posséderait un caractère purement déclaratif de l'intention qui s'y trouve exprimée.

Cette représentation de l'interprétation comme œuvre de pure déduction dans tous les cas est, de nos jours, rejetée par la plupart des juristes.» ${ }^{19}$

Pourtant, la déduction (en particulier sous le nom de «syllogisme») demeure largement utilisée par les rédacteurs des jugements. Il y a là une dimension rhétorique de très grande importance, de l'ordre de la justification. En d'autres termes, on se trouve ici face à la mise en formes ou Présentation d'une solution dont on dispose antérieurement, solution qui a été élaborée pour une très large part en dehors du syllogisme. Insistons: la déduction «exploite ce qui est déjà là ${ }^{20}$ (typique de l'analytique); elle est «de l'ordre de la preuve $»^{21}$.

Le philosophe du droit belge Rigaux rend compte, à l'aide de formules provocantes et d'une extrême sévérité, de l'esprit de la Présentation et son enracinement dans une conception du monde selon l'univocité: «Les praticiens [...] ont tendance à déguiser leur activité sous des formules qui en masquent le ressort. En réponse à cette obscurité savamment entretenue, le présent ouvrage se voudrait une mise au jour des principes sur lesquels se fonde le raisonnement judiciaire.

18 J. Ray, cité par M. Van de Kerchove/F. Ost, Le système juridique entre ordre et désordre, PUF, Paris, 1988, 73.

19 J.-P. Côté, «L'interprétation de la loi. Une création sujette à des contraintes », in Lire le droit. Langue, texte, cognition sous la direction de D. Bourcier et P. Mackay, LGDJ, Paris, 1992, 145 avec les références à la doctrine anglo-saxonne.

20 J.-B. Grize, «Les raisonnements semi-formels », in Lire le droit. Langue, texte, cognition sous la direction de D. Bourcier et P. Mackay, LGDJ, Paris, 1992, 107.

21 J.-B. Grize, «Déduction et inférence», in Lire le droit. Langue, texte, cognition sous la direction de D. Bourcier et P. Mackay, LGDJ, Paris, 1992, 233. 
On peut citer, parmi les vaches sacrées de la philosophie spontanée des juristes, la recherche du sens littéral, la théorie de l'acte clair, le devoir du juge d'obéir à la loi, la distinction entre le fait et le droit [...], l'idée que pour toute question litigieuse il n'existerait qu'une seule solution correcte. Ces lieux communs de la pensée juridique participent à une taxinomie binaire, c'est-à-dire à une méthode de raisonnement traçant une démarcation rigide entre la face positive et la face négative de toute réalité. Toutefois, la multiplicité des règles ayant vocation à appréhender les faits complique ce que les juristes voudraient spontanément pouvoir tenir pour une opération logique de qualification. ${ }^{22}$

La constatation du pouvoir d'invention ou pouvoir créateur du juge va de pair avec la reconnaissance de l'incidence de la pragmatique, de l'importance du contexte et de la culture des «interprètes autorisés ${ }^{23}$ ainsi que de leurs expériences et de leurs convictions personnelles. On est alors très loin de la pureté logique (logique formelle), de l'objectivité exigée par le positivisme juridique.

Transparaît au travers de la critique de Rigaux, sur le mode d'une alternative, une autre signification de regula, celle de régularité, une règle sur le mode du «le plus souvent» ou «le plus fréquent» selon la conception aristotélicienne, laquelle, par ce gradient, laisse apparaître la possibilité d'une conception gradualiste de la catégorie légale par opposition à sa nature binaire, discrète, homogène (Chapitre 4).

Revenant à la conception orthodoxe de la catégorisation juridique, on relèvera que le concept légal devient la seule mesure du cas et l'évaluation une stricte recherche de conformité au sens de correspondance trait pour trait. Le genre absorbe de la sorte toutes les espèces, tout ce qui en elles pourrait l'excéder devenant non-pertinent pour leur qualification en tant qu'occurrence de ce type: les espèce sont conformes au concept légal (genre) ou ne sont rien pour le droit. Le cas ne s'y trouve donc jamais considéré en lui-même c'est-à-dire selon ses singularités mais n'est pertinent qu'en les éléments identiques aux traits de la catégorie légale qu'il recèle. A l'exprimer en stricte logique, le cas ne donne aucunement lieu à jugement en justice, à recherche de juste, puisque le juste qu'il pourrait contenir ou auquel il devrait mener lui vient exclusivement de la catégorie légale (ou genre), seule apte à enregistrer le juste déposé en elle ensuite de l'arbitrage de valeurs opéré par le législateur. Le cas se contente, via la «con-formité », de répéter ce juste légal, ce qui empêche de rendre justice au cas particulier comme tel et dans ce cas-ci, attitude allant à l'encontre même de la justice, du juste, du jus c'est-à-dire du droit selon l'étymologie latine. Faut-il rappeler la médiocre place ménagée à l'équité, à peine considérée dans sa médiation infra legem (dans le cadre de la loi) et déconsidérée dans ses interventions praeter legem et contra legem comme l'indiquent les expressions elles-mêmes: praeter, à côté de (la loi) et contra, contre (la loi), soient dans tous les cas à la marge.

En d'autres termes, la comparaison générique et son critère de la conformité, effaçant toute la singularité du particulier, contreviennent à la notion même de justice suivant ce paradoxe qui traverse le droit moderne: il se dit formellement jus

22 F. Rigaux, La loi des juges, Odile Jacob, Paris, 1997, 8.

23 Expression de J. Chevallier, «Les interprètes du droit», in Interprétation et droit, sous la direction de P. Amselek, Bruylant, Bruxelles, 1995, 115 ss., en particulier 120 à 122 «La communauté des interprètes». 
(en latin), à savoir, normalement, juste (d'où justice), jus traduit par «droit», tout en acceptant d'être injuste matériellement, sur le fond, le cas échéant ${ }^{24}$, en tant qu'il recevrait son contenu de manière parfaitement passive du législateur, aveuglement volontaire construit grâce à une focalisation sur la seule forme de la loi.

La forme de la loi recouvre ici aussi bien le respect des procédures d'adoption des textes législatifs (la «bonne» forme) que la non-pertinence de la finalité (rien que la forme ${ }^{25}$ ) ou but de la loi - et plus globalement sa part d'équité qui devrait en être l'âme au même titre que l'ordre - comme condition de sa «juridicité». Si tout le droit se ramasse dans la seule loi et que de celle-ci n'est exigé qu'un respect des procédures préétablies, alors le strict légalisme permettra aux droits totalitaires, comme le droit national-socialiste, d'être formellement du droit et aux juristes de les appliquer en toute «bonne conscience juridique» car en parfaite cohérence avec la théorie de référence, théorie pure du droit.

Cette cohérence s'avère à la forme extrêmement solide, servant l'une des exigences les plus fondamentales d'un système de droit: la sécurité juridique. Mais là encore prévaut une conception formelle de la sécurité juridique. Or, la sécurité juridique peut être conçue de manière dynamique sans pour autant perdre la dimension de prévisibilité de cette légitime exigence de tout ordre juridique. La sécurité juridique s'éprouve infiniment plus labile que ce que donnent à voir ses contours extérieurs, conduisant souvent à la confondre avec quelque chose de statique, une sorte de mécanisme voire de mécanicisme inspiré de la physique moderne. Pareille souplesse et labilité de la sécurité juridique n'en ruine aucunement la dignité, pas plus que l'efficacité. Elle nous oblige simplement à changer de paradigme, plus exactement d'exhumer la conception sémiotique qui toujours fut à l'œuvre dans la pratique du droit mais que la Présentation du droit sous une forme syllogistique notamment (par opposition à son Effectuation) - ou Représentation - masquait. L'esquisse d'une conception dynamique de la sécurité juridique peut être élaborée à partir de ou au sein d'un domaine prima facie des plus retors à une appréhension dynamique de ce ressort de l'ordre juridique: le formalisme notarial. Et pourtant, «Qu'est-ce qu'un acte notarié à côté de la demeure où l'on habite? Comment comparer cette fragile feuille de papier à l'épaisseur des murs et des mémoires? Aucun rapport de ressemblance, aucun mimétisme, aucune référence, aucun plan. Et pourtant, en cas de conflit, en cas d'héritage, en cas de dispute, c'est bien par le lien foudroyant de cette feuille dérisoire avec le corpus des textes, par l'intermédiaire des avocats et des juges, que je pourrai prouver, authentifier mon bien - et conserver ma maison. L'attache est minuscule et pourtant totale; l'emprise infime et pourtant capable de se lier à tout le reste.» ${ }^{26} \mathrm{La}$ question de la sécurité juridique est bien davantage affaire de traces, d'esquisser un chemin dans le réseau de signes juridiques, tracé le plus probable eu égard au droit positif et à la phase du système judiciaire concernée.

${ }^{24}$ Par exemple dans le cas du droit national-socialiste.

25 D'un point de vue philosophique, pour le légalisme, la forme (eidos) de la loi n'est pas «seulement» le signe de la fin, du but, expression de sa cause finale, mais la fin elle-même, formule de $\mathrm{M}$. Bastit, Naissance de la loi moderne, PUF, Paris, 1990, 49, capturant d'un coup tout l'idéalisme (Idée, forme ou eidos) du positivisme juridique ainsi que le ressort des théories procédurales contemporaines de la justice, du juste, de la démocratie.

26 B. Latour, La fabrique du droit, La Découverte, Paris, 2002, 284. 
Aussi sommes-nous invités à dépasser la vision du droit selon le «légalisme » et de la sécurité juridique selon la «con-formité», participant tous deux de la réduction de principe du droit à la seule loi, laquelle parlerait d'elle-même, tour d'esprit consacré par l'expression «la loi dit que...», déjà mentionnée, présupposant un sens déposé de toute éternité dans le texte.

Le droit contemporain brise en effet cet image d'Epinal d'un droit semblant se complaire dans un hiératisme le rendant rapidement inapplicable compte tenu de la multiplication incessante des relations et des activités humaines, des changements de valeurs subséquents, de l'accroissement des conflits des normes juridiques entre elles ainsi qu'avec les règles morales, situation d'hétérogénéité favorisant d'autant l'intervention de l'équité, marque contemporaine du droit ${ }^{27}$.

On comprend d'autant mieux cette description de Schwind: «Pour le droit même comme système de règles normatives cette évolution signifie un changement profond. Les règles normatives dans leur forme extérieure sont invariables comme les termes qui les expriment. Mais ces formes ne sont que des capsules dont le contenu est variable sans qu'on s'en aperçoive. La capsule reste invariée jusqu'au moment où le contenu a tellement changé que la capsule éclate et on doit en trouver une autre qui s'adapte mieux à la fonction changée.

Le système juridique entier, considéré par la plupart de la doctrine comme une structure presque fortifiée qu'on peut interpréter mais qu'on ne peut pas changer, est mis en cause. L'invariabilité du système est plus apparente que réelle. Les termes invariés n'indiquent point que le contenu et la fonction sont encore les mêmes qu'ils étaient au moment de la création desdits termes.» ${ }^{28}$

La pratique du droit, l'application de la loi aux occurrences, rétroagit donc sur la signification de la catégorie légale, cette dernière ne se contentant pas d'absorber les cas: elle en «subit» l'influence, la rétroaction.

Tenter d'élaborer la théorie de la pratique du droit et non la théorie de l'Idée $d u$ droit exige, notamment, de changer le ressort du droit, abandonner la «comparaison générique » pour une «comparaison prototypique » conçue telle que chaque occurrence modifie la catégorie légale qui l'assume.

\section{LA COMPARAISON PROTOTYPIQUE, UNE CONNAISSANCE BOTTOM-DOWN OU SELON L'ANALOGIE}

\section{Un juste omniprésent, en filigrane}

Nous avons débuté cet article par des considérations juridiques hautement techniques et formalistes: le calcul des délais. Nous avons vu que cette technicité, ce formalisme ne sont pas des fins en soi mais, au rebours, de simples moyens au

${ }^{27}$ Lequel requiert davantage de confiance en le juge, de par la trop grande difficulté de trouver des solutions justes dès le stade de la loi dans nos sociétés toujours plus éclatées: «Il est plus difficile de légiférer pour «inclure» que pour exclure: le polythéisme des valeurs et la conciliation des intérêts divergents n'autorisent désormais que des normes dont l'indécision implique une délégation à l'interprète.», P. Martens, Théories du droit et pensée juridique contemporaine, Larcier, Bruxelles, 2003, 197.

28 F. Schwind, «Aspects et sens du droit international privé», in Recueil des Cours de l'Académie de droit international, Sijthoff, Leyde, 1984, IV, 9 ss, 125-126. 
service du juste ou de la justice. Notion fortement décriée alors qu'elle fonde cette autre, de tous reconnue, voire révérée: l'interdiction de l'arbitraire. Le Tribunal fédéral n'articule-t-il pas en effet la notion d'arbitraire autour de celles d'équité et de justice? «Une décision est arbitraire lorsqu'elle viole gravement une norme ou un principe juridique clair et indiscuté, ou lorsqu'elle contredit d'une manière choquante le sentiment de la justice et de l'équité.» ${ }^{29}$

Pourquoi tant d'hésitation, de retenue face aux notions de justice ou d'équité? Parce qu'elles seraient vagues, exagérément vagues? N'en va-t-il pas ainsi de toutes les figures fondamentales du droit: le principe de confiance, la bonne foi, le modèle du bon père de famille (bonus pater familias), l'étalon du comportement diligent ou encore «selon les circonstances du cas», quand ce ne sont pas les recours innombrables dans les textes législatifs à l'analogie... figure nodale de la pensée humaine, pensons-nous, mais sans définition juridique pourtant $\mathrm{t}^{30}$.

Plus déroutant, la vacuité des grandes notions du droit, à tout le moins leur amphibologie, semble atteindre le ressort même de l'activité de ces hommes du livre - du code - que sont les juristes (les civil lawyers), rompus à toutes les techniques d'interprétation des textes. Il n'est pas jusqu'à la méthode la plus parfaite, la plus incontestable parce que parfaitement univoque, la méthode dite «littérale» qui ne soit frappée de la même ambiguïté. En effet, elle n'échappe pas non plus à des considérations de justice alors même qu'on la conçoit généralement comme objective, donnant lieu à une pure opération formelle de décodage suivant une logique bijective. A la faveur d'une décision de 2006, le Tribunal fédéral a eu l'occasion de préciser sa démarche en matière d'interprétation, affirmant qu'il «ne privilégie aucune méthode d'interprétation, mais s'inspire d'un pluralisme pragmatique pour rechercher le sens véritable de la norme ${ }^{31}$. Sans discuter ici la notion des plus controversées en linguistique et en sémiotique de «sens véritable», on soulignera l'affirmation de la Haute cour de procéder pragmatiquement, attitude qui la pousse à admettre que le fond sur lequel se détache tout raisonnement juridique, toute interprétation textuelle, consiste en le juste; ainsi poursuit-elle, «en particulier, il ne se fonde sur la compréhension littérale du texte que s'il en découle sans ambiguïté une solution matériellement juste [+références citées]» $\gg^{32}$.

29 SJ (Semaine judiciaire) 2001 I 466.

30 Parmi des dizaines de milliers d'occurrences, signalons le Règlement d'exécution (vaudois) de la loi fédérale sur la faune, art. 61 «Chasse sur le lac Léman en bateau», dont l'al.3 porte «Est considéré comme une embarcation tout bateau, radeau ou engin analogue [nous soulignons], qu'il soit amarré, ancré, ou non.»

31 Arrêt du 13 février 2006, 4C.296/2005.

32 Ce qui signifie entre autres que le «texte clair» c'est-à-dire l'inutilité de recourir à l'interprétation du moment qu'un texte livre par lui-même son sens (comment, puisque l'on a sous les yeux que de l'encre, du papier, tous deux liés en des formes que l'on dénomme alphabet, si l'on reconnaît un système de signe qui nous soit connu ou auquel on puisse le ramener), - suivant l'adage latin claris non fit interpretatio -, n'est jamais que le raccourci cognitivo-rhétorique de l'équité qui n'apparaîtra pas expressément s'il la réalise. En d'autres termes, l'interprétation peut être dite littérale et, partant apparaître seule (sans considération de contenu, de justice), que si elle emporte une solution matériellement juste. A cette aune, le formalisme n'est que d'apparence, manière de Présentation du droit, et plus spécifiquement du juste, lequel se révèle de l'ordre de l'Effectuation du droit, ce dans quoi il se réalise, se «par-fait», exerce son essence. 
Nous n'aborderons pas non plus les rapports entre le juste et l'équité, les liens qu'ils entretiennent ne prêtant guère à un doute de principe, sauf à admettre un juste «conformité» (supra Chapitre 3 ), lequel vide la notion de juste de tout contenu, même vague, même «sentimental», pour se ramasser dans la formule «juste parce que prescrit».

Or, pour vagues que soient ces notions, plus précisément du fait même qu'elles sont ambiguës, elles appellent par quelque nécessité interne la référence à des cas exemplaires, que l'on se gardera de lire suivant la subsomption ou classement «genre/espèces», qui nous interdirait de pénétrer plus avant le principe, en demeurant sur le plan de sa seule généralité plutôt que de tenter d'en esquisser la direction cognitive, d'en exprimer les indices de sens. Simplement «indices» puisque la littéralité elle-même n'est juridiquement recevable qu'en tant qu'elle « est» (en vérité qu'on la peut construire comme) consonante à une solution matériellement juste. Les cas font donc vivre le principe, le «substantialisent», l'érigent. Exprimé par l'autre extrémité de la relation, le principe se sédimente au fil des cas, s'y définit et s'y densifie. Ainsi s'avère-t-il trop unilatéral, statique, d'affirmer que les occurrences sont des applications du principe, ce qui sous-entend qu'il a déjà quelque contenu spécifique, ce qui est certainement vrai d'un point de vue diachronique mais tout à fait insuffisant en tant que le principe par nature opère in concreto : il se nourrit des cas qu'il structure et qui l'informent en retour suivant des rétroactions. Un principe n'est en rien un point-origine, un point sans dimension, sans épaisseur, sans culture mais s'éprouve tout au contraire pétri de diachronie, résultat toujours passager de traditions, d'habitudes de pensée, de tour d'esprit propre à une certaine société.

Tout type d'inférence linéaire, qu'il soit inductif ou déductif, s'avère impropre, de par sa complexion lisse, sans boucle (de rétroaction), à saisir la dynamique reliant le principe aux cas. Les logiques du développement (au sens géométrique du terme) ou du déploiement sont trop analytiques (au sens du jugement analytique de Kant, par opposition au jugement synthétique) pour rendre raison de ce que le principe reçoit son contenu des cas «d'application »: il ne recèle pas toujours déjà tous les arbitrages que les juges opéreront in concreto au nom du principe. Au mieux y opère-t-il sous le mode de la directive, à la manière d'un but vaguement esquissé, un sentiment dont il faut échafauder les raisons d'y adhérer, les arguments de s'en convaincre, conduisant l'esprit aux cas, remontant sans plus attendre de ces concrets à l'abstrait de l'orientation suggérée, suivant un mouvement de va-et-vient, mode d'inférence dénommé abduction (Paragraphe 2). En bref, le principe en appelle par nature aux cas (Paragraphe 3).

\section{Au nom de l'abduction - compendium de ce mode d'inférence rétroactif. Ou, de l'analogie déguisée}

La notion d'abduction étant peu connue, il convient de l'aborder par des exemples. Après ce propédeutique par «exemplarité», nous esquisserons quelques définitions et descriptions de l'abduction pour en circonscrire les traits fondamentaux et l'originalité de pensée qu'elle porte, en particulier en plaçant au centre des démarches cognitives la comparaison, non la comparaison générique, on s'en doutera, mais la comparaison prototypique, procédant bottom-down. 
L'abduction est un (type de) raisonnement par analogie permettant de conquérir l'inconnu à partir du connu. Soit un sujet connaissant les oiseaux et les ailes qui en assument la motricité dans les airs. Son regard portant pour la première fois sur un poisson en train de nager, il forgera ou pourra forger l'hypothèse, par analogie avec le rapport connu «ailes/oiseau sous la raison de motricité », donc par inférence bottom-up, que ce dont bat le poisson et dénommé nageoire assure au poisson, dans un milieu différent, une fonction analogue de motricité à celle réalisée par des ailes d'oiseau, établissant le rapport nageoire/poisson sous la même «raison » ou série de rapports (de proportions, soit «analogie» au sens génuine): fonction motrice $=$ ailes $/$ oiseau $=$ nageoire $/$ poisson .

Le processus des découvertes scientifiques révèle des jeux d'analogies très élaborés où s'entremêlent ressemblances et dissemblances, à l'instar de la démarche de Kepler cherchant à résoudre le problème des orbites planétaires, plus exactement de trouver une solution à un cas surprenant: «Kepler a appris de ses prédécesseurs que les orbites des planètes sont circulaires. Puis il observe deux positions de Mars et remarque qu'elles touchent deux points (x et y) qui ne peuvent être les deux points d'un même cercle. Le cas est curieux. Il ne le serait plus si l'on assumait que les planètes décrivent une orbite représentée par un autre type de courbe et si l'on vérifiait que x et y sont deux points de ce type de courbe (non circulaire). Kepler doit donc trouver une loi différente. Il pourrait imaginer que les orbites des planètes sont paraboliques ou sinusoïdales... Il importe peu ici de savoir pourquoi il pense à l'ellipse (il a sans doute de bonnes raisons). Il établit donc son Abduction: si les orbites des planètes étaient elliptiques et si les deux positions de Mars révélées (x et y) étaient un Cas de cette Loi, le Résultat ne serait plus surprenant. Naturellement, il lui faut à ce stade vérifier son Abduction en feignant une nouvelle Déduction [un troisième point, z, de l'ellipse, position prédite et vérifiée]» ${ }^{33}$.

La conquête de l'inconnu par une analogie avec le connu renvoie à la présence englobante, voire surplombante, de la culture laquelle, si elle peut être mise plus aisément à distance (semble-t-il) en sciences «dures », pénètre au plus profond les sciences humaines. C'est dans l'épaisseur de la culture que s'originent les réflexions humaines sur le savoir et les pratiques comme l'exprime si ouvertement l'éthique, venant d' «êthos «manière d'être habituelle, caractère » et «mœurs »» à suivre le Dictionnaire historique de la langue française, Le Robert, Paris, 1992, verbo éthique, 738-739. Vision tout aussi pragmatique glanée à une autre source, le Dictionnaire de la langue française lexis, Larousse, Paris, 1988, verbo éthos, 686: «Caractère commun à un groupe d'individus appartenant à une même société».

Dès lors, pour en revenir à l'élection par Kepler de l'orbite elliptique, on ne s'étonnera pas qu' «il eut à choisir entre diverses courbes géométriques, dont le nombre n'était pas infini, et les thèses précédentes [nous soulignons] sur la régularité de l'univers l'amenèrent à ne chercher que des courbes fermées ${ }^{34}$.

La description de cette découverte scientifique livre nombre d'enseignements parmi lesquels nous ne retiendrons que ceux ayant trait à l'analogie (et par là à

\footnotetext{
33 Eco, De Superman au Surhomme, Grasset, Paris, 1993, 181 [cité Superman].

34 Eco, Les limites de l'interprétation, note 4, 264.
} 
l'abduction). Les deux modes classiques de l'analogie y apparaissent intimement intriqués. D'une part, l'analogie de proportionnalité, soit le type $\mathrm{a} / \mathrm{b}=\mathrm{c} / \mathrm{d}$, comme moteur de l'intelligence opérant par comparaison en référence à un premier rapport connu, dans notre cas la «motricité aile/oiseau », érigé en point de départ de l'acte de connaissance et non point-origine. Ce point de départ est lui-même porté, d'autre part, par une analogie d'attribution - second mode de la figure de l'analogie - par laquelle on attribue au rapport inconnu quelques ressemblances du rapport connu afin de l'apprivoiser, de se le rendre familier.

Ainsi conquerrait-on jamais l'inconnu qu'à partir du connu. Comment, en effet, pourrait-on appréhender le «parfaitement» différent, le radicalement incommensurable? L'analogie de proportionnalité se retrouve de la sorte au cœur épistémologique de l'acquisition du savoir, précisément de par sa structure proportionnelle.

Admettant qu'on ne conquiert l'inconnu qu'à partir de quelque connu pris pour référence, la pensée s'engage de la sorte bien plus profondément que dans une « simple» comparaison ou, plus platement encore, que suivant un simple classement de l'espèce nouvelle sous le genre - comment ce dernier est-il su adéquat? Elle procède par abduction, au sens d'invention d'une hypothèse: «un rapport étant connu, un terme tiers «surprenant» se présente, que l'on tente d'apprivoiser c'est-à-dire auquel on tente de s'habituer en le rapportant partiellement à un habitus cognitif, un rapport déjà connu, une connaissance sédimentée dans la culture dont dispose le sujet avant même que ne lui apparaisse le cas surprenant. On se rappellera Kepler ayant appris de ses prédécesseurs que les orbites des planètes sont circulaires (fermées); subissant l'attraction de ce «connu» (manifestement premier connu pour lui), il optera, dans la solution de l'orbite de Mars, pour un «cercle à deux centres », à savoir l'ellipse, laquelle ne répondait plus exactement aux propriétés du cercle.» ${ }^{35}$

Ce raisonnement complexe articulé autour de l'analogie n'a rien d'un choix arbitraire de quelques affinités entre des objets parfaitement disparates par ailleurs. Ce raisonnement par analogie, cette première approximation de l'abduction, se déploye selon un processus inférentiel se distinguant aussi bien de la déduction que de l'induction. Comme annoncé, de ce qu'elle consiste en une «[F]orme de raisonnement encore peu reconnue ${ }^{36}$, nous en présenterons plusieurs définitions.

Nous tirons du Superman de Eco une seconde définition, plus honnêtement approximation: «Si j'ai un Résultat curieux dans un champ de phénomènes non encore étudié, je ne peux chercher une Loi de ce champ (si elle existait et si je la connaissais, le phénomène ne serait pas curieux). Je dois aller «enlever» ou «emprunter» [abduction en anglais] une loi ailleurs. Si vous voulez, je dois raisonner par analogie.» ${ }^{37}$ La richesse de la notion apparaît explicitement. Son appartenance à une épistémologie voire à une métaphysique de l'analogie se peut comprendre par référence à cette phrase inaugurale d'Aristote «l'être se dit multiplement» que Eco interprète en sémioticien-épistémologue: le philosophe

\footnotetext{
35 A. Papaux, op. cit. note 12, 177.

36 D. Durand, La Systémique, QSJ, PUF, Paris, 1994, 98.

37 Eco, Superman, 182.
} 
grec «pose les conditions mêmes du discours qui permet d'aborder des phénomènes dissemblables de façon unitaire [...] Le philosophe essaie de poser un concept qui permette d'interpréter globalement une série de phénomènes [...] Le philosophe ne découvre pas la substance, il en pose le concept. Le jour où le scientifique découvre qu'avec la dialectique substance-accident il ne réussit plus à expliquer les nouveaux phénomènes qu'il détermine, il n'infirme pas une hypothèse scientifique, il change simplement de critères épistémologiques, il refuse une métaphysique influente. $\rangle^{38}$

Ce tour d'esprit n'a rien d'excentrique mais constitue tout au contraire un procédé usuel, pas toujours conscient sans doute, mais bien présent dans la démarche philosophique et plus globalement dans la cognition générale. Ricœur procédera de cette manière dans l'un de ses derniers ouvrages Parcours de la reconnaissance: «Cet essai est né d'un pari [pari qui est au cœur de l'abduction verronsnous], celui de conférer à la suite des occurrences philosophiques connues du mot «reconnaissance » la cohérence d'une polysémie réglée » ${ }^{39}$. Une polysémie réglée soit un champ sémantico-pragmatique (suivant un sens sémiotique cherchant à tenir ensemble l'occurrence et le type) tissé de ressemblances et de dissemblances de significations, structure de «même » et de «autre» mêlés, intriqués, hybride en lequel on reconnaît la figure de l'analogie.

Le «ailleurs» de «..."emprunter" une loi ailleurs...» de la citation de Superman traduit la puissance transgénérique de l'analogie, autorisant le passage d'un genre à un autre, d'un champ de connaissance à un autre. En cette propriété épistémologique de conquête de l'inconnu par l'analogie, se retrouve l'abduction dans sa dimension cognitive soit, en termes kantiens, l'anticipation, d'un jugement synthétique, certes à vérifier par un processus d'essai/erreur, au rebours de la déduction toujours analytique, allant toujours du même au même, du point de vue du genre à tout le moins.

Au reste, l'abduction demeure étrangère à la certitude absolue, ce que montre Eco en rappelant la finitude des facultés intellectives humaines: «Si je vois sur une table une assiette remplie de thon en conserve et à côté une boîte de thon, ouverte et vide, l'hypothèse conséquente est presqu'automatique: mais c'est ce presque qui fait de ce raisonnement automatique encore une hypothèse. ${ }^{40}$

L'abduction condense les formes de la tentative et de l'invention, elle les tient ensemble et simultanément : elle participe du pari et de l'anticipation. On peut lors en proposer une définition supplémentaire: «une méthode pour former une prédiction générale sans assurance positive qu'elle réussira dans un cas particulier ou d'ordinaire, sa justification étant qu'elle est le seul espoir possible de régler rationnellement notre conduite future, et que l'induction fondée sur l'expérience passée nous encourage fort à espérer qu'un jour elle réussira.» ${ }^{41}$

L'«espoir» auquel il est fait mention dans cette citation assoit la nature hypothétique du processus abductif, lequel n'est jamais qu'une «tentative hasardée,

38 Eco, Sémiotique et philosophie du langage, PUF, Paris, 1988, 12-13 [cité Sémiotique].

39 P. Ricœur, Parcours de la reconnaissance, Stock, Paris, 2004, 10.

40 Eco, Les limites de l'interprétation, op. cit., 260.

${ }^{41}$ Les Notions Philosophiques, verbo abduction, in Encyclopédie philosophique universelle, 2 tomes, sous la direction de S. Auroux, PUF, Paris, 1990, 4, reprenant la définition de Peirce. 
d'un système de règles de signification à la lumière desquelles un signe acquerra son propre signifié.» ${ }^{42}$ Par la nécessaire présence d'un code (au sens large de réseau de significations), ne serait-ce point le culturel qui s'insinue dans la logique de la découverte scientifique, médiation dont voulait justement nous affranchir les démarches inductive et déductive?

L'abduction révèle en ce point la nature singulière de la métaphysique et de l'épistémologie qui l'autorisent: l'acte de connaissance dont elle essaie de rendre raison doit se comprendre comme jeu simultané d'un Sujet et d'un Objet: «si la comparaison requiert des «faits», notamment le Résultat surprenant et les éléments d'un premier rapport connu, il y a aussi «invention» d'une hypothèse, loi ou Règle, telle que la compréhension du Résultat comme Cas de la Loi le lave d'une singularité - le «surprenant»-dont l'assomption en tant que tel excède les puissances de la science. La part active du sujet dans l'acte de connaissance - présupposée dans la définition même de l'abduction en ce qu'elle ne traduit pas une inférence nécessaire - s'incarne dans la «tradition» (en un sens tout générique) dont l'efficace revêt la forme de «cadre de référence», de «règle métalinguistique ${ }^{43}$. Inscrit dans une tradition - on se souviendra des éthos scientifiques et extra-scientifiques, d'un Kuhn par exemple -, le sujet connaissant ne s'identifie aucunement à quelque Sujet transcendantal, situé au-dessus des contingences du monde, dans une pure synchronie l'affranchissant de toutes les influences de la société hic et nunc. Eco adopte un point de vue résolument réaliste en relevant l'importance des indices circonstanciels et contextuels dans l'invention de l'hypothèse $\mathrm{e}^{44} \gg^{45}$. Ainsi assume-t-il la force inamissible des cas dans la connaissance, laissant entrevoir que l'acte de connaissance n'est pas séparable d'une casuistique.

\section{Droit, cas «exemplaire» et règle du précédent}

Le juriste peut-il dans la pratique se passer des cas, des exemples, des manières de leading cases ou encore Schulbeispiele? En d'autres termes, une pratique juridique est-elle envisageable sans une casuistique; la peut-on concevoir sur le seul mode de la subsomption, du classement genre/espèces et, partant de la comparaison générique?

En ouverture, nous avons abordé la loi sur l'aide au victime d'infractions (LAVI) prévoyant la substitution de l'Etat à l'auteur d'un crime ou délit en vue de la réparation du préjudice physique et moral subi par la victime.

Or, à peine se dispose-t-on à exercer la loi, à l'appliquer, que les exemples nous viennent nécessairement à l'esprit, «nécessairement» en ce que l'on ne peut faire autrement, cela s'impose, quand bien même aurait-on voulu en rester à la formulation générale et abstraite de la loi. La loi, rien que la loi, fût-ce toute la loi, ne semble pas faire sens.

\footnotetext{
42 Eco, Sémiotique, 51.

43 Eco, Sémiotique, 50; voir aussi 51.

44 Eco, Sémiotique, 51.

45 Papaux, op. cit. note 12, 186.
} 
Par exemple, une certaine gravité de l'atteinte est exigée pour une aide LAVI à la victime. Mais que peut bien signifier une atteinte d'une certaine gravité? La loi demeure silencieuse... et comment pourrait-elle faire autrement? Ne faudraitil pas détenir des tabelles au nombre d'entrées quasi infini afin de rendre justice à chaque cas particulier dans sa singularité même, sous l'angle de la gravité, de «sa» gravité? N'est-ce point là pourtant la simple expression de la justice, du juste, soit du droit? La loi se dévorerait elle-même, aboutissant à cette situation aussi paradoxale que funeste: un cas, une loi (une entrée dans le répertoire). La loi cesse d'être générale et abstraite et, partant, cesse d'être «loi ».

La pratique est nettement plus pragmatique que le modèle juridique traditionnel «subsomption + syllogisme + genre/espèces ». Elle opère à partir et par des exemples, des scenarii-types, des social patterns sédimentés dans la culture juridique des juristes au cours d'une très longue formation universitaire et professionnelle. La doctrine joue un rôle fondamental d'élaboration du corpus d'exemples-types, de scenarii-modèles qui donnent chair aux catégories légales générales et abstraites, sans lesquels elles demeureraient trop éloignées des cas pour les régir ou alors donneraient dans l'arbitraire épistémologique dans leur passage vers les cas singuliers et concrets. Comment en effet éviter cet arbitraire épistémologique face au saut qualitatif "général et abstrait vs singulier et concret» puisque le syllogisme, la déduction ou encore la subsomption, ne procèdent que du même au même, sont «homo-gènes » donc relevant du même genre, inaptes dès lors à rendre raison du franchissement de ce saut qualitatif.

Ni le choix de la majeure du syllogisme, ni la détermination de la «différence spécifique » du classement «genre/espèces», ne sont pas explicités, ni même théorisés dans la description classique du raisonnement juridique. La qualification juridique y est simplement décrite comme subsomption mais non pas expliquée, ni même explicitée comme le relève Blanchard: «Lorsqu'il [le processus de la qualification] se donne à voir, c'est sous la forme déjà achevée de la qualification » ${ }^{46}$.

L'abduction, la dialectique type-occurrences et la médiation des exemples, des scenarii-modèles se situent précisément à cet endroit.

Ainsi, Mizel, commentant la LAVI, abordant des catégories légales donc générales et abstraites, livre-t-il immédiatement des exemples ou des modèles, non pas simplement pour les illustrer mais pour les «signifier», pour qu'elles puissent faire sens et non demeurer dans des formules creuses, nécessairement creuses, plus exactement a priori, soit « a priori causa » donc sans besoin d'en passer par l'expérience c'est-à-dire par des cas, réels ou prototypiques. Mizel explicite de la sorte le ressort de telle catégorie légale, le référent pratique, mieux pragmatique, qui en permettra l'exercice dans les cas concrets: «Une certaine gravité de l'atteinte est exigée, comme par exemple une invalidité ou une diminution durable d'un organe important $»^{47}$. Il en va de même pour le Tribunal fédéral concernant les atteintes à

46 F. Blanchard, «Vers une théorie de la qualification juridique. Les socles épistémiques de la catégorisation ", in Lire le droit. Langue, texte, cognition sous la direction de D. Bourcier et P. Mackay, LGDJ, Paris, 1992, 223 ss., 226.

47 C. Mizel, «La qualité de victime LAVI et la mesure actuelle des droits qui en découlent », in JT [Journal des tribunaux] $2003 \mathrm{IV}, 38$ ss. 96 (les soulignés sont de nous) + les références qui multiplient les «par exemple» comme «...des circonstances traumatisantes, par exemple avec des gaz lacrymogènes.» 
l'intégrité psychique lesquelles «n'entrent en considération pour une réparation morale que lorsqu'elles sont importantes, telles des situations de stress post-traumatiques qui aboutissent à une modification durable de la personnalité ${ }^{48}$.

On dispose de la sorte des prototypes, soient de référents ou premiers rapports connus pour élaborer les analogies de proportion et conquérir l'inconnu porté par les cas nouveaux.

Est-ce à dire qu'il existerait une règle du précédent dans le droit continental (civil law), une démarche juridique qui procéderait de cas en cas, apanage traditionnel, suivant la théorie juridique légaliste, du common law dit justement «case law»?

Des civilistes l'affirment, dès lors que le regard porte sur l'exercice de la loi, sa fin ultime selon nous, c'est-à-dire son application...aux cas. Gaudemet ${ }^{49}$ affirme la «souscription implicite (ou expresse) de tout système juridictionnel à la règle du précédent». Rigaux relève que «Au sens large, le positivisme juridique inclut les décisions des tribunaux. Il reconnaît la force des précédents judiciaires, qui sont une variante de l'argument d'analogie: si la situation soumise au tribunal est voisine d'un cas déjà tranché, le juge doit se rallier à la solution antérieure ${ }^{50}$.

Où l'on retrouve une démarche de nature circulaire ou dialectique, annonçant la rétroactivité du cas sur la loi, de la situation de «fait» sur la catégorie légale ou encore, pour le formuler dans les termes de la sémiotique comprenant l'abduction, de l'occurrence sur le type (légal). La règle du précédent signifie alors que la rétroaction est elle-même médiatisée par le référentiel épistémologique et cognitif que constitue le «prototype» ou «occurrence exemplaire», lequel permet d'élaborer l'analogie de proportion sur base d'un premier rapport connu qui consiste en un social pattern, un "scénario social-modèle».

Semblable tour d'esprit enjoint d'abandonner les «méthodes » d'interprétation juridique au profit d'une véritable herméneutique ${ }^{51}$, changement de paradigme que l'on peut schématiser par la substitution d'une dialectique (type-occurrences), d'une démarche rétroactive du cas vers les lois - soient des procédés dynamiques - par opposition à des protocoles statiques, des méthodes si fixes qu'elles en deviennent «recettes», tout pénétrés de la comparaison générique recherchant la correspondance trait pour trait entre cas et loi sur le mode de la «con-formité».

Ricœur a de fort belle façon caractérisé ce changement de paradigme, désignant pour cœur de la démarche juridique d'application le raisonnement par analogie, sous la forme du jugement des ressemblances et des dissemblances, marquant de la sorte implicitement le retour de l'ars juris, d'un savoir-faire, donc d'une expérience, d'une tradition, d'une culture, par contraste avec une Rechtswissenschaft: "L'application d'une règle juridique est en fait une opération très complexe où l'interprétation des faits et celle de la norme se conditionnent mutuellement. Du côté des faits de la cause en procès, le même enchaînement factuel peut être interprété de plusieurs manières [...]; et telle façon d'en lire

48 Cité par Mizel, op. cit., 97 (les soulignés sont de nous).

49 Y. Gaudemet, «Fonction interprétative et fonction législative: aménagements juridiques de leurs rapports », in Interprétation et droit, sous la direction de P. Amselek, Bruylant, Bruxelles, 1995, 201 ss., 204.

50 F. Rigaux, op. cit, 128-129.

51 Sur ce changement de paradigme, voir notamment A. Papaux, op. cit. note 12, $161 \mathrm{ss.}$ 
l'enchaînement est déjà orientée par la présomption selon laquelle tel enchaînement narratif place le cas considéré sous telle règle de droit. Quant à la règle de droit, elle présente elle-même une open structure qui la met sous la dépendance des interprétations antérieures adoptées dans des cas semblables; mais l'idée même de cas semblables, présupposée par la règle du précédent ${ }^{52}$, résulte de l'interprétation du degré de ressemblance ou de dissemblance avec les cas précédents. [...] Cela dit, il n'y a plus rien de scandaleux à avouer le caractère vague du langage juridique, les conflits entre normes, le silence de la loi sur les cas inédits, la nécessité de choisir entre la lettre et l'esprit de la loi : c'est en l'appliquant que non seulement on reconnaît la norme comme contraignante, mais que l'on fait l'épreuve de sa variabilité, et que s'exerce le jeu décrit plus haut de l'interprétation mutuelle entre la loi et les faits.» ${ }^{53}$

Se découvre une démarche circulaire ou rétroactive, l'esquisse d'un raisonnement bottom-down, soit de cas par rapport (au sens premier de rapporté à) à un autre cas, ce dernier exemplaire ou prototypique de la catégorie légale envisagée. Cette catégorie est envisagée dans un premier temps comme simple hypothèse possible en vue de l'exhaussement du cas litigieux à l'existence légale, avant que d'être confirmée dans son statut de catégorie légale (et non plus de simple hypothèse) pour la qualification «définitive» du cas sous réserve d'acceptabilité.

«L'interprétation de la loi, pour être appliquée dans un cas d'espèce, doit être considérée comme une hypothèse, que l'on adoptera, en définitive, que si la solution concrète à laquelle elle aboutit s'avère comme acceptable. C'est uniquement en tenant compte de cette double exigence, qui nécessite un va-et-vient de l'esprit, de la situation vécue à la loi applicable, que l'on comprendra la spécificité de la pensée juridique.» ${ }^{54}$

A la faveur de l'acceptabilité, réapparaît la texture profonde, intime du droit: l'équité et donc le sentiment du juste, dussions-nous être interdit d'en pouvoir donner une définition ferme, définitive. N'est-ce point là au reste l'enseignement le plus grave des dialogues de Platon, que Deleuze présentait comme paradoxal: mal, mouvement, devenir ne peuvent être saisis en eux-mêmes, conduisant à « conclure de manière ouverte »: seule la justice et juste et la vertu vertueuse, laissant pleine place au jugement et à l'exemplarité. Aristote me sombrera pas plus dans la tautologie lorsqu'il définira, en première ligne, la sagesse comme l'œuvre du sage.

\section{Esquisse d'un modèle de comparaison bottom-down}

La dénomination «bottom-down» sans doute est-elle des plus singulières. Elle veut simplement exprimer que le cas à résoudre est moins général, pour être tout

52 Y. Gaudemet, op. cit., 204, parle de «souscription implicite (ou expresse) de tout système juridictionnel à la règle du précédent », en laquelle se fonde l'autorité de l'interprétation du juge. La règle du précédent n'opère donc pas dans les seuls pays de common law. Dans le même sens, Rigaux, op. cit., 129.

53 P. Ricœur, «Le problème de la liberté de l'interprète en herméneutique générale et en herméneutique juridique », in Interprétation et droit, sous la direction de P. Amselek, Bruylant, Bruxelles, 1995, 177 ss., 180.

${ }^{54}$ Ch. Perelman, Logique juridique. Nouvelle rhétorique, Dalloz, Paris, 1979, 83. 
à fait exact moins générique ou matriciel, que le prototype. Le prototype, en effet, a été rendu en quelque manière abstrait de par sa fonction d'exemplarité: il mène le jeu - leading (case) -, le jeu de l'analogie de proportionnalité. Il est bien cas, mais cas-référence.

La possibilité, et même l'évidence, de l'inadéquation de la lettre de la loi à sa portée réelle - reconnue par les non-juristes aussi bien - dévoile une logique prototypique (ou dialectique typeloccurrences) à l'œuvre dans la compréhensioninterprétation des textes juridiques. L'interprétation littérale ne se révèle telle qu' en fonction d'un certain contexte culturel ${ }^{55}$ qui assigne à l'occurrence retenue par le législateur une valeur exemplaire ou prototypique, comme dans le cas d'une pancarte disposant que «les chiens sont interdits sur les perrons de la gare». Le philosophe du droit belge, Ch. Perelman, initiateur de l'Ecole de Bruxelles interroge: comment jugera-t-on un voyageur tenant en laisse un ours apprivoisé?

Appliquant la méthode d'interprétation dite littérale, chacun sera convaincu que la signification de la phrase ne prête pas à doute, au point qu'aucune interprétation n'est nécessaire: un chien est un chien; il n'a rien à voir, du point de vue de la taxinomie scientifique, avec l'ours, la baleine ou la tortue géante. En toute cohérence, ces derniers animaux seront donc autorisés! Conclusion logiquement irréprochable... mais très peu vraisemblable néanmoins.

Pourquoi ce hiatus entre la logique et la vraisemblance pratique, l'expérience voire l'expérimentation pragmatique? Il exprime justement la présence d'un audelà des mots, d'un au-delà des «significations immédiates», le dépassement d'une plate sémantique. Pour l'exprimer «littéralement», ici chacun peut «voir» les mots fabuler, raconter des histoires ou fabula, porter des scripts, ramasser des frames, sédimenter des scenarii.

Compte tenu de ces derniers, juristes comme non-juristes soutiendront que l'interdiction ne se limite pas aux canidés. La «lettre» de la loi ne s'éprouve pertinente pour personne, dans cette culture, en tant que les appréciations relatives à la portée de la signification de «chien» ne procèdent aucunement d'un arbre de Porphyre, d'une logique binaire: l'ours entrera dans le cercle des animaux interdits dans la gare, aussi bien que la chèvre, la vache, l'orang-outan, alors que la tortue géante ou la baleine ne viennent pas même à l'esprit. Les travaux préparatoires ne changeront rien à l'affaire puisqu'en toute vraisemblance, aucun de ces animaux n'a retenu l'attention «du» législateur.

Pourquoi ce dernier ne les aurait-il pas même envisagés? La réponse est d'ordre culturel: aucun ne ressortit des scenarii habituellement déposés dans la mémoire des ressortissants de cette culture à propos des animaux se pouvant trouver sur les quais d'une gare et des types corrélatifs de danger qu'ils y incarnent; plus spécifiquement, aucun ne suit les schémas habituels de la pensée juridique suisse, pas plus qu'européenne au reste.

Cette interprétation est confirmée par le fait, avéré, que le profane ou nonjuriste comprend sans grande difficulté la portée de l'interdiction au-delà de «chien», au-delà de la lettre de l'inscription, compréhension juste et du juste d'autant plus édifiante que, par hypothèse, le profane n'a pas été formé aux

55 Outre la justice matérielle à réaliser dans le cas «malgré » la lettre de la loi applicable, voir la décision du TF mentionnée en matière d'interprétation littérale. 
subtilités de l'interprétation juridique. C'est dire que la question posée par la pancarte, et de là sa signification, n'est pas en première ligne juridique. Plus exactement, le droit qui a pour souci principal sa commensurabilité aux pratiques sociales réelles ou potentielles, table sur une compréhension de la lettre de la loi qui ne suive pas les taxinomies scientifiques mais, tout au contraire, s'appuie sur des connaissances «communes», des expériences acquises par éducation, par exercice de la vie collective et ses représentations dans telle société et par l'imitation, toutes pratiques indispensables pour agir dans la cité, pour fonder des actions efficaces, bref pour accomplir ses finalités, son destin d'homme, de zoon politikon, d' «animal politique». La «traversée» de la lettre de la loi vers son esprit devient possible de par l'expérience de la vie commune, des social patterns.

Cette référence, implicite, aux expériences du monde, aux mœurs, à la doxa (opinion commune), aux usages, à toutes ces pratiques en théorie extérieures au texte légal, n'en demeure pas moins parfaitement connue et envisagée in casu c'est-à-dire dans le cas de la pancarte: elle fait partie de la ratio legis, de la «raison» de la loi.

On se gardera toutefois d'oublier que la considération de la fin, de la finalité, la «lecture» téléologique du monde (ici de la loi), fut longtemps disqualifiée, à la faveur du grand rêve, quand ce n'est pas à proprement parler du mythe, de la Rechtswissenschaft. A cette aune, les considérations téléologiques ne pouvaient qu'apparaître «métaphysiques», idées abstraites voire éthérées entourant toute réflexion relative au but (telos). La disqualification (relative) de l'approche «téléologique» du réel s'affiche sans ambages dans la sphère juridique où la méthode d'interprétation dite «téléologique» n'est jamais qu'affirmée ultima ratio: la dernière «méthode» à laquelle recourir, la moins fiable, la plus sujette à caution, celle qui expressément charrie des jugements de valeurs, à l'encontre de la scientificité du droit telle que l'imaginaient les épigones d'une Rechtswissenschaft.

L'exemple de la pancarte ne fait qu'illustrer cette mise à l'écart ou relégation du but, de la finalité. L'attention au but ne s'éprouverait-elle point trop sensible aux contextes, à la pragmatique, voire aux préférences de l'interprète? Ne s'exposerait-on pas à perdre la règle comme regula, régularité, somme toute comme étalon permettant de dire le droit - il s'agirait pourtant moins de «dire le juste» que de répéter la loi -, d'arrêter la bonne mesure?

Cette réserve ne fait sens qu'à la condition de lire le droit selon le paradigme de la «con-formité», d'une mimétique trait pour trait à dresser, mieux à reconnaître, entre le cas litigieux et la catégorie légale générale et abstraite.

En revanche, considérer la fin, le but de la loi, et plus largement la finalité du droit (le juste, l'équité) dont la loi n'est qu'un raccourci cognitif, conduit à un changement de perspective dans l'appréhension des réalités: une lecture selon la fonction, privilégiant le «pour-quoi » et donc la ratio legis, se substitue à la lecture selon la structure entendue ensemble de propriétés analytiques.

La compréhension-interprétation du «chien» mentionné sur la pancarte ne cherche plus à mimer à l'identique la description du chien telle qu'on la peut trouver dans les dictionnaires, à en partager trait pour trait les propriétés analytiques, singulièrement «plates», voire insignifiantes pour le cas à traiter: «Animal domestique dont il existe de nombreuses races ayant diverses aptitudes: chasse, garde de troupeaux ou de maison...» dispose le Larousse Lexis, entrée 
«chien». Devait-on s'en remettre au seul sens du dictionnaire suivant la doctrine du «sens ordinaire» ou «sens naturel» des mots encore souvent retenue par la dogmatique juridique? Combien il serait difficile de savoir quel est l'objet de l'interdiction et son «pour-quoi », alors même que tout un chacun, le locuteur «ordinaire » en particulier, sait à quoi s'en tenir dès la prise de connaissance du texte de la pancarte. Partant, dans les cas les plus fréquents, soit à nouveau la regula-régularité, standard d'un comportement attendu du citoyen "moyen », selon une représentation sociale induite de la société considérée, rares seront qui viendront ours en laisse ou vache à la main, de surcroît convaincus qu'ils seraient en cela dans leur «bon droit». Par où l'on mesure combien la lecture de la définition du dictionnaire, sans l'expérience des social patterns de chien, des scenarii concrets de comportements de chien tels que les a enregistrés la culture concernée par photo, film, peluche, bandes dessinées, mythes et autres comptines, etc., est artificielle, contrefactuelle même et assurément inadéquate au comportement attendu de ce citoyen «standard», lequel pourtant se comportera bien selon une interprétation du chien comme fonction, «dans les cas les plus fréquents» évidemment ${ }^{56}$.

On obtient de la sorte un mode de compréhension de la pancarte plus réaliste, davantage fidèle à l'acte de «lecture» linguistique comme juridique du texte légal, esprit passant d'une vision analytique du réel à une conception réticulaire ou en réseau de significations, de fonctions. On passe du «chien» en tant que «chien» à «chien » comme vecteur d'une certaine fonction juridique ou prototype d'un certain scénario ou script, ayant servi ou servant de référentiel pour l'élaboration du sens de la norme, in casu la fonction (ratio legis) «fluidité du trafic», «risque accru d'accident ferroviaire», «hygiène » ou encore «importunité des utilisateurs », etc.

Au rebours de la logique de l'univocité, ici prévaut la complexion, la texture, matricielle des «mots »: au lieu de lire «chien» comme l'étiquette désignant toutes les occurrences de l'espèce zoologique concernée, l'interprétation selon la fonction ou l'«analogie cognitive», entend «chien» comme modèle, schéma, prototype, représentant emblématique, «exemplaire», sorte de standard du type d'animal dont la présence sur un perron de gare pose problème eu égard à la ratio legis. Au vu de cette dernière, l'ours se comprendra comme «chien» selon une analogie de fonction; mêmement pour la vache. Semblable démarche requiert d'avoir circonscrit le contexte d'application du texte en cause, considération qui remet par ailleurs en cause l'existence en droit de «textes clairs», transparents en soi. Par exemple, dans le contexte de l'Inde, pour quelque temps encore, affronté à une vache sacrée, nul n'osera la contraindre à quitter les lieux, quel que soit le danger créé, son statut d' «animal sacré » interdisant de l'assimiler à un trivial «chien».

L'interprétation-qualification des réalités par comparaison bottom-down en référence à une occurrence prototypique, «représentative» - «chien» dans notre exemple - permet de saisir le phénomène juridique dans son mouvement même

56 Rappelons combien fut contre-productif l'usage des chiens dans la recherche des terroristes en Irak, l'armée américaine remontant contre elle une grande part de la population en tant que leur demeure se retrouvait souillée par la présence de ces animaux, aucunement porteurs d'impureté pour un occidental. 
via la dynamisation de l'intension ${ }^{57}$ (ou compréhension) de l'entité considérée, laquelle ne se déduit plus de quelque essence éternelle ou «nature des choses» univoque mais s'infère des fonctions, des social patterns, scenarii que peut endosser cette entité dans les contextes considérés comme les plus fréquents dans la culture donnée. Toute entité en tant qu'elle porte la possibilité de se couler dans l'un des social pattern consacré par tel système juridique est à même de retenir l'attention du juriste: elles deviennent pour lui pertinentes dès lors qu'elles «jouent» une fabula ${ }^{58}$ juridique. C'est pourquoi l'éducation du juriste se trouve en grande part consacrée à l'apprentissage des ces fabula, expressions de la ratio legis.

Ces fabula ou scripts ou scenarii constituent l'un des ressorts de la comparaison bottom-down, esquissant l'orientation que les analogies cognitives auront à suivre en référence au scénario-modèle (prototype) pour être reçues comme analogies pertinentes en droit, à défaut de quoi elles pourraient bien passer pour de vagues ressemblances le devant à l'arbitraire bien davantage qu'à quelque cohérence rhizomique ou de système. Par où la comparaison bottom-down prend un tour résolument pragmatique, concret comme dans le cas Rylands v. Fletcher à la faveur duquel l' "électricité » fut assimilée, soit rendue similaire à, donc analogue à, l'«eau »: «Pour découvrir si le principe de Rylands v. Fletcher peut s'appliquer à une affaire de fuite d'électricité (escape of electricity) qui cause le dommage, il faut se demander si l'électricité se révèle analogique à l'eau, car c'est celle-ci qui a occasionné le dommage dans Rylands et par conséquent l'eau est, par rapport à l'électricité, un fait pertinent.» ${ }^{59}$

\section{CONCLUSION}

La démarche par comparaison bottom-down répond au projet général d'embrasser le droit dans sa pratique, de tenter de le montrer en acte, dans son application, et non plus d'en rester sur le seul plan de la loi, légalisme ou law in books, plan de l'Idée du droit par oubli ou négligence du droit pratiqué, prononcé, du «droit dit», celui de la «juris-dictio», le «dire-le-droit».

Adoptant la perspective de la «pratique du droit $»^{60}$, la nature concrète de la fabula apparaît en pleine lumière, par contraste saisissant avec les classifications scientifiques, souvent abstraites pour le commun des mortels: la présence d'un chien dans une gare et les scenarii qu'elle induit dans l'esprit des ressortissants de

57 Ou, en termes philosophiques, la compréhension du concept (en gros, ses propriétés, ses composants), par opposition à son extension (l'ensemble des cas qu'il recouvre).

$58 \mathrm{Au}$ sens large de fabula: une séquence d'actions (Eco, Superman, 14) qui «raconte» donc quelque chose de la vie.

59 G. Samuel, «Entre les mots et les choses : les raisonnements et les méthodes en tant que sources du droit », in Revue internationale de droit comparé, 1995, 2, 513-514 En bref, la question pertinente devient: en termes de dommages, l'«électricité » raconte-t-elle la même (selon la similitude) histoire que l'«eau»? Où l'on reconnaît la narrativisation de l'interrogation se substituant à une enquête conduite en termes exclusivement logiques sous l'égide du critère de la «con-formité» ou identité traits à traits du cas et de l'hypothèse légale (assumée par le prototype).

${ }^{60}$ A strictement penser, l'expression constitue un oxymore: le droit est une praxis, sa destination génuine consistant à rendre justice dans le cas singulier soit le cas pratique. 
la culture considérée se laissent plus aisément représenter que «membre de la famille des canidés». La fabula est plus «parlante» par le fait d'intégrer un ou plusieurs contextes, généralement parmi les plus profondément sédimentés dans la culture considérée.

La théorie (de la pratique) du droit connaît ces histoires privilégiées et les reconnaît sous les dénominations de Schulbeispiele, de cas d'école ou encore, plus emblématique, de leading cases. Elles portent ou supportent la règle du précédent, ressort de l'adaptation du droit aux changements des pratiques sociales et des représentations collectives. Elles explicitent le premier rapport connu de l'analogie de proportionnalité, exhibent la relation de référence par rapport à laquelle sera élaboré le raisonnement, «par analogie » précisément, dont découlera la solution au cas litigieux. L'électricité n'est pas l'eau mais dans le contexte d'un détournement ou d'une responsabilité pour dommage, leur qualité similaire de «fluide» justifie d'appliquer, analogiquement, à la première ce que les juges avaient élaboré pour la seconde en matière de de «ponctions sauvages » ou encore à propos des dommages causés.

La catégorie légale entendue comme fabula se développe suivant une logique davantage horizontale que verticale - telle qu'illustrée par la subsomption - dont le référentiel n'est autre que le prototype de la catégorie légale. Le «proto-type», par définition «à la place [proto-] du type», ramasse, sur un mode mnémotechnique, le scénario, le script le plus représentatif de la catégorie, le plus exemplaire pour le contexte et la culture donnés, à l'instar de «chien» pour présence animale (présumée faire problème) sur un quai de gare.

Sur base de cet exemple prototypique, l'interprète peut élaborer une comparaison avec le cas litigieux; il recherche les ressemblances, ce faisant découvre des dissemblances et procède à une pesée des unes et des autres afin de décider, véritablement de juger c'est-à-dire d'apprécier, d'évaluer, s'il y a ou non une analogie juridiquement pertinente entre les deux fabula. Dans l'exemple de la pancarte, entre «chien» sous-entendu prototypique et loup: oui; entre «chien» et vache: oui; entre «chien » et écureuil: non; etc. En bref, «le jugement procède non plus d'un raisonnement déductif mais de la comparaison [horizontalité de la démarche] avec une moyenne selon la technique du standard», mettant en œuvre «une «règle de jugement» au sens d'une mesure moyenne de conduite sociale $»^{61}$.

Le caractère pragmatique, concret du scénario légal - par contraste avec la classification strictement scientifique donc analytique et dichotomique, fondée sur l'univoque - permet de franchir le premier pas dans le sens de la commensurabilité à construire ( $c f$. Ricœur, supra) entre la loi générale et abstraite et le cas singulier et concret. En ce sens, la proposition théorique d'une qualification juridique par comparaison bottom-down rend plus adéquatement raison, fût-ce toujours imparfaitement, de la pratique du droit.

Est-il besoin de rappeler que « la loi humaine toujours à mi-chemin du général qu'elle vient préciser, et du particulier qu'elle ne peut rejoindre sauf à perdre son

${ }^{61}$ D. Salas, «L'équité ou la part maudite du juge», in Justices. Revue générale de droit processuel, Dalloz, Paris, $\mathrm{N}^{\circ}$ 9, 1998, 113. 
essence de loi, est en effet un instrument imparfait ${ }^{62}$ ? Intrinsèquement imparfait rappelait Aristote, dès lors sans qu'il puisse en être tenu rigueur d'aucune manière au législateur. Le juste, soit le droit au sens le plus accompli, attend son achèvement, sa «per-fection » (d'être réalisé de «part en part), du juge dans son « dire-ledroit» («juris-dictio»).

Et si l'analogie avec le cas-référence, le prototype, se construit, si la commensurabilité du cas, singulier et concret, avec la loi, générale et abstraite, s'élabore, alors le raisonnement juridique se coule tout entier dans le bain de l'équité, éther pense-t-on volontiers, éminemment concret, pragmatique au contraire pour qui attache son regard au cas plutôt qu'à la loi ou, plus artificiellement encore, au texte de la loi: "Quant au mécanisme de l'équité, ensuite, la solution du juge ne résulte pas spontanément du vague sentiment de ce qui est juste dans la cas dont il est saisi, sentiment de pitié ou de charité. L'équité est un étalon, un instrument de mesure; il s'agit toujours de comparer entre deux termes, entre deux intérêts, intérêts individuels le plus souvent [...]. Concilier, composer, l'équité est toujours une œuvre de pesée. La balance est son symbole, la proportionnalité son critère. [...] Qui ne voit alors qu'en réalité, l'équité n'est pas une dimension exceptionnelle de l'office du juge; c'est son principe même.» ${ }^{63}$

Faculté de droit

Université de Lausanne

62 M.-F. Renoux-Zagamé, «'Royaume de la loi’: équité et rigueur du droit selon la doctrine des parlements de la monarchie», in Justices. Revue générale de droit processuel, Dalloz, Paris, $\mathrm{N}^{\circ}$ 9, 1998,36

63 L. Cadiet, «L'équité dans l'office du juge civil», in Justices. Revue générale de droit processuel, Dalloz, Paris, $\mathrm{N}^{\circ}$ 9, 1998, 95. 